Cultures \& Conflits

56 | hiver 2004

Militaires et sécurité intérieure.

\title{
Imaginaire du contrôle des foules dans l'armée de terre française
}

\section{Elwis Potier}

\section{OpenEdition}

1 Journals

\section{Édition électronique}

URL : http://journals.openedition.org/conflits/1609

DOI : 10.4000/conflits.1609

ISSN : $1777-5345$

Éditeur :

CCLS - Centre d'études sur les conflits lilberté et sécurité, L'Harmattan

\section{Édition imprimée}

Date de publication : 1 décembre 2004

Pagination : 35-49

ISBN : 2-7475-7598-X

ISSN : 1157-996X

Référence électronique

Elwis Potier, «Imaginaire du contrôle des foules dans l'armée de terre française », Cultures \& Conflits [En ligne], 56 | hiver 2004, mis en ligne le 07 janvier 2010, consulté le 30 mars 2021. URL : http:// journals.openedition.org/conflits/1609; DOI : https://doi.org/10.4000/conflits.1609

Ce document a été généré automatiquement le 30 mars 2021.

Creative Commons License 


\title{
Imaginaire du contrôle des foules dans l'armée de terre française
}

\author{
Elwis Potier
}

There are in fact no masses; there are only ways of seeing people as masses. Raymond Williams ${ }^{1}$

1 Certaines images fortes perçues avec une vive émotion imprègnent notre mémoire pendant un temps, parfois toute une vie. Par la suite, la vue d'une image similaire va réactiver toute cette émotion nous amenant à voir l'ancienne image comme si elle était présente à nouveau. Il en va de même pour certains phénomènes sociaux qui marquent l'imagerie sociale à tel point que les acteurs en situation reproduisent les mêmes réactions passionnelles qu'à l'époque du moment déclencheur².

2 A peine avions-nous fermé les yeux sur les « mouvements de foule » qui ont ébranlé la France à la fin du XIX ${ }^{\text {ème }}$ siècle et au début du XX ${ }^{\text {ème }}$, donnant naissance à la fois dans le champ des sciences humaines à la psychologie collective et dans le champ politique aux techniques de propagande ${ }^{3}$, qu'ils se sont rouverts sur les populations étrangères se soulevant contre les forces armées, et en premier lieu contre celles des colonisateurs, après la seconde Guerre Mondiale, notamment lors de la guerre d'indépendance de l'Algérie. Sur le territoire national, les foules de l'intérieur se sont peu à peu institutionnalisées, grâce notamment aux réglementations issues de la crise de $1934^{4}$, pour devenir des manifestations : rassemblements organisés, ritualisés, cadrés par les manifestants eux-mêmes et par des forces de l'ordre spécialement pourvues à cet effet (CRS et gendarmerie mobile). On peut ainsi définir le maintien de l'ordre, en ce qu'il est différencié du contrôle militaire des foules, non seulement, cela va de soi, par l'ordre social préétablit qu'il est censé « maintenir » et les attributions des forces de l'ordre qui en ont la charge, mais aussi et surtout par l'objet même qui le mobilise, à savoir la « manifestation ».

Dans ce cadre social intérieur où l'ordre institué permet la circulation de "masses » manifestantes, la foule est toujours là - les observateurs en parlent ou la font parler derrière les différents rassemblements ou mouvements de la population (des 
populations) mais son statut a changé : elle n'est plus personnifiée, elle n'est plus Une. Cette réunion particulière d'individus qu'est la foule, relayée aux confins de l'espace public, est redevenue anonyme ${ }^{5}$; elle a perdu de son unité et donc de sa puissance symbolique, diluée qu'elle est désormais dans les mouvements aux abords des stades ou dans un public nombreux venu contempler un spectacle. Pour autant la foule, entité à part entière, existante par elle-même et pour elle-même, celle dont on croit voir la véritable nature se dévoiler en même temps qu'elle se donne en spectacle, cette foule-là revient à la faveur de l'actualité, par le débat autour de l'exercice des fonctions de police par les forces armées françaises. Elle revient aujourd'hui par l'extérieur, en dehors de nos frontières, parmi les populations étrangères faisant l'objet d'interventions sous mandats internationaux, sous l'égide de l'ONU. Ces populations se retrouvent confrontées, outre les belligérants engagés dans les combats, à d'autres militaires venus non pas pour faire la guerre mais disent-ils pour rétablir ou maintenir la paix.

Depuis la fin de la Guerre Froide et le bouleversement mondial des rapports de force qui s'en est suivi, l'armée de terre française est en pleine refondation ${ }^{6}$. Prenant acte de la rupture stratégique provoquée par la nouvelle donne géopolitique, pour faire face aux nouvelles crises internationales comme celle du Kosovo, l'armée de terre française a dû redéfinir sa doctrine de façon plus réaliste, considérant qu'il n'était " plus réaliste d'ignorer la présence de civils et de non-combattants sur les théâtres d'opération » ${ }^{7}$. C'est dans ce contexte et suivant ce mode de légitimation que les militaires français vont produire un discours spécifique désignant sous l'appellation " contrôle des foules » une pratique instituée, codifiée et vouée à s'étendre, dont l'objet central «les foules » pose pour le moins question.

Quelle que soit la raison d'être de l'intervention, qu'il s'agisse d'une opération de guerre, de soutien à la paix, de sécurité ou de secours d'urgence, les forces terrestres peuvent être appelées à contrôler les foules qui feraient «obstacle » à leur mission. Le discours officiel diffusé dans les écrits de doctrine militaire présente ces foules comme de "nouveaux acteurs" des théâtres d'opération, lors des engagements en dehors du territoire national ${ }^{8}$. Le langage militaire les définit en conséquence et avant tout par leur violence intrinsèque fondée sur la crainte qu'elles suscitent ${ }^{9}$. Cette crainte peut prendre une forme singulière, que certains relient à l'agoraphobie ${ }^{10}$, renouant avec la peur séculaire $\mathrm{du}$ nombre faisant masse ${ }^{11}$ toujours latente depuis l'avènement des «foules révolutionnaires ${ }^{12}$. Les foules, violentes par nature, retrouvent une caractérisation propre, un caractère physique évident, mais également de façon plus prégnante un caractère psychologique, et vont se décliner suivant une typologie exclusivement construite sur la violence et le degré de menace qu'elles inspirent. On établit alors un continuum, une graduation dans la violence qui va de la manifestation paisible à l'insurrection, indiquant par le fait la progression possible, "naturelle", d'une foule générique indépendamment du contexte dans lequel elle se meut. Les types de foules deviennent rapidement des stades d'évolution, et l'on peut s'attendre à tout moment au passage d'un état à un autre. Mais, s'il convient de maîtriser les débordements possibles, de prévenir les éventuelles émeutes ou insurrections, d'où vient ce besoin de contrôler précisément cette forme, singulière s'il en est, que l'on nomme foule? Qu'est-ce qui sous-tend ce discours greffé sur ce vieux mot apparemment anodin et pourtant si évocateur? 
6 La question se pose au vu des nouveaux enjeux stratégiques et politiques desquels a émergé cette rhétorique instituée sur les foules. Les militaires spécialistes de la question ne manqueront pas d'en appeler au "réalisme » et de rappeler, exemples à l'appui, l'effectivité des mouvements de foules qu'ils rencontrent sur le terrain. Pour eux, parce qu'ils y sont confrontés, les foules existent bel et bien: insurrections, débordements, pillages, manifestations, etc., peu importe les mots, la chose existe et il faut y faire face. Leur préoccupation est de savoir comment réagir, avec quels moyens, quelles procédures et suivant quels objectifs. Cependant, cette réalité, affirmée et réaffirmée, posée d'autorité comme irréfutable, ne préjuge en rien de l'emprise des significations imaginaires sur les perceptions du réel: "Le sujet est dominé par un imaginaire vécu comme plus réel que le réel, quoique non su comme tel, précisément parce que non su comme tel $»^{13}$. La question de l'imaginaire s'impose nécessairement à quiconque s'interroge un tant soit peut sur le sens même du mot foule et sa définition. L'équivoque dans laquelle le mot est maintenu - il est notoire que le mot connote plus qu'il ne dénote - rend sa signification très aléatoire, malléable, manipulable et somme toute très peu scientifique ${ }^{14}$. L'analyse des usages du mot à travers les textes de doctrine, les articles traitant de la question et des pratiques y compris dans leurs aspects les plus techniques aboutit à soulever la question de la dimension imaginaire qui façonne les représentations. A tous les niveaux, de la théorie à la pratique, le contrôle des foule est, tel qu'il nous est donné à voir et à entendre, directement connecté à l'imaginaire de la foule issu de la littérature et principalement de la psychologie des foules ${ }^{15}$. Un fond commun réunit ces idées développées sur les foules, un humus imaginaire dans lequel s'enracinent pour partie conceptions et pratiques.

7 Il nous faut emprunter cette voie parfois sinueuse de l'imaginaire afin de rendre compte des significations qu'il donne à la pensée de l'institution (l'Armée française en l'occurrence) : il s'agit de comprendre comment l'imaginaire se présente et participe d'une certaine réalité, celle des foules. Est-il besoin de préciser que nous ne proposerons ici qu'une ébauche, quelques pistes qui pourraient se poursuivre ailleurs, autrement. Pour autant, on ne saurait prétendre entamer une réflexion sur cette question difficile et complexe de l'imaginaire sans en expliciter les référents théoriques. Enonçant le postulat de «l'immanence essentielle du sens à la pratique $»^{16}$, nous tenons pour indissociable la relation entre sens et action. La pratique du contrôle des foules, considérée comme telle et donc aussi comme dialectique, devra être prise pour une "activité dans laquelle les significations sont impliquées et constitutives ${ }^{17}$. Pour cette raison, notre ligne directrice sera essentiellement tracée par les significations imaginaires sociales telles qu'elles ont été conceptualisées par Cornelius Castoriadis. Notre investigation s'inscrit dans cette pensée qui comprend l'imaginaire comme "substantif $»^{18}$ et s'appuie en conséquence sur les catégories fondamentales du philosophe. Posons simplement "qu'il y a des significations relativement indépendantes des signifiants qui les portent, et qui jouent un rôle dans le choix et dans l'organisation de ces signifiants. Ces significations peuvent correspondre au perçu, au rationnel, ou à l'imaginaire $»^{19}$. Ajoutons que les significations imaginaires sociales sont premières en ce sens qu'elles« orientent » le fonctionnel et le symbolique ${ }^{20}$. Si l'imaginaire chez Castoriadis renvoie d'avantage à l'instituant, à l'imaginaire radical, il n'en reste pas moins vrai que toute recherche doit partir des faits et donc du réel toujours déjà institué afin d'élucider les significations imaginaires de l'institution. Nous tenterons donc de dégager ces significations à l'œuvre dans l'imaginaire institué par l'armée française autour de cette notion propre à l'armée de terre qu'est le contrôle des foules. 
8 La maîtrise de la violence, nouvelle thématique qui accompagne désormais la traditionnelle coercition de forces, fournit le cadre au sein duquel le contrôle des foules se veut incontournable. Ce serait un moyen nécessaire, indispensable même pour certains, entendu qu'il ne relève pas seulement de la tactique, c'est aussi une affaire de stratégie. Maîtriser la violence, comme objectif et comme stratégie ${ }^{21}$, constitue le cadre à l'intérieur duquel s'inscrit le contrôle des foules dont la justification prend en compte les deux positions d'attaque et de défense "pour protéger les populations face aux belligérants ou protéger les unités de nos forces face aux populations. Même si les forces terrestres n'ont pas pour mandat de maintenir l'ordre public, la maîtrise des mouvements de foules peut participer à l'effort de maîtrise de la violence $»^{22}$.

9 La visée globale de l'action, sur le plan stratégique, amenant à la confrontation avec ce qui est décrit à travers une terminologie appartenant au registre des phénomènes de foules, va induire une identification instantanée entre ces mouvements et la foule ellemême. Les foules seront désormais considérées comme des acteurs, avec des comportements et même des intentions ${ }^{23}$. La personnalisation parachève le processus qui mène de l'observation de certains phénomènes dits de foules à leur édification (ou réification) en tant qu' "acteurs". Mais le contrôle ne peut être qu'extérieur, la régulation ne peut provenir de la foule elle-même. C'est parce qu'un groupe humain faisant masse, d'où la métaphore physique également contenue dans l'étymologie du mot foule, est d'abord perçu comme une chose (menaçante) à maîtriser, avant de pouvoir être pensé comme une "personne collective", que la foule n'est définie et pensée que dans une position en dehors, par l'observateur ou l'acteur extérieur à cette chose dont il ne peut (ou ne doit pas) faire partie, et ce en fonction de son statut, de ses impressions et de sa volonté. La volonté de maîtrise (ou de contrôle) se focalise sur cet objet mal délimité, flou, et néanmoins reconnu d'emblée comme foule sans le comprendre de l'intérieur, sans prendre en compte sa situation, ce qui a pour effet de décontextualiser les phénomènes évoqués, de les détacher de leur ancrage culturel, social et historique, où on voit l'effet de l'essentialisme véhiculé par ce discours qui reprend de la sorte de façon plus ou moins implicite l'hypothèse de la «foule psychologique » de Gustave Le Bon ${ }^{24}$. Par cette opération forcément réductrice, le mot foule condense et met en équivalence des ensembles humains de types très différents pour créer une nature ad hoc, une essence dont on verra par la suite le sens. La foule est tantôt une partie de la population, tantôt la population dans son entier, le peuple ${ }^{25}$, mais elle peut tout aussi bien être un rassemblement, un défilé, une manifestation festive ou de révolte, une assemblée, un électorat, etc.

10 Le discours porté sur les foules dans le "contrôle des foules", de la théorie la plus officialisée par les textes de doctrine à la pratique sur le terrain en mission de type OPEX (Opérations Extérieures) s'arrange bien du flou entretenu par un usage immodéré, pour ne pas dire incontrôlé, du terme souvent confondu avec celui de masse. Le glissement délibéré de la foule vers la masse marque ce que l'on pourrait repérer comme le "moment» décisif à partir duquel peut se structurer tout un imaginaire de la foule, vue à la fois comme émanation et symbole ${ }^{26}$ de la population ou du peuple. On retrouve ce passage d'un terme à l'autre dans les textes de doctrine (TTA 950 ) et dans la plupart des ouvrages consacrés à la question ${ }^{27}$. La référence aux théories de Gustave Le Bon, ainsi qu'à celles d'Elias Canetti ${ }^{28}$ pour ce qui est des masses, même si elle n'est pas toujours explicite ${ }^{29}$, n'en est pas moins omniprésente et explique en grande partie d'où proviennent ces conceptions. 
11 Dans cette optique, la foule peut alors désigner à peu près tout et n'importe quoi, à la seule condition qu'il s'agisse d'un groupe humain que l'on souhaite contrôler. Tout groupe - le terme de groupe est à la fois plus large et plus neutre - peut être désigné et par la même stigmatisé comme foule s'il interfère de façon négative dans l'action engagée. La géométrie variable des contours qui sont censés délimiter la notion de foule, afin de pouvoir en faire un objet assez distinct pour justifier d'un traitement à part, d'une technique particulière, a pour fonction de l'insérer dans les paramètres miliaires et d'exercer sur elle un contrôle opérant. La volonté de faire de la foule un objet d'expérience, impliquant de possibles « retours d'expérience », est d'abord mue par la nécessité de la maîtriser, ce qui aboutit à travers la réification, la condensation et la confusion sémantique (avec la «masse ») à l'attribution d'une personnalité fictive, personnalisation qui puise largement dans les stéréotypes de l'imaginaire collectif.

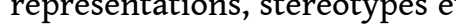
autres clichés sur les foules qui circulent depuis si longtemps et dont les traits principaux se sont figés à la fin du $19^{\mathrm{ème}}$ siècle. Impossible de comprendre ce qui est en jeu dans la persistance du fond imaginaire de cette époque sans en identifier la source intellectuelle et idéologique ${ }^{30}$. Il nous faut donc remonter jusqu'à la «foule psychologique ", expression que Le Bon va propager à partir de 1895. A cette date paraît son fameux ouvrage Psychologie des foules, livre qui aura le succès que l'on sait et qui sera apprécié de nombreux grands chefs d'Etat et chefs militaires ${ }^{31}$.

13 L'influence des théories leboniennes dans l'armée s'explique d'abord par l'insertion personnelle de Le Bon au sein du commandement militaire français dans les deux décennies précédant la deuxième Guerre Mondiale ${ }^{32}$. Rejeté par les milieux universitaires et scientifiques ${ }^{33}$, Le Bon en savant éclectique et opportuniste, trouve un accueil enthousiaste de la part de plusieurs «savants maîtres » de l'Ecole de guerre dont le général Bonnal et le colonel de Maud'huy."Ce sera peut-être par l'armée que notre Université subira la transformation qu'elle refuse d'accepter " prophétise-t-il en espérant ainsi enfin acquérir la reconnaissance tant attendue ${ }^{34}$. Son apologie de l'esprit militaire, sinon de la guerre elle-même, lui aura permis d'exercer une influence considérable auprès de l'état-major français : "L'esprit militaire constitue la dernière colonne soutenant les sociétés modernes et, pour cette raison mériterait la reconnaissance des peuples qui le maudissent $\aleph^{35}$. Notons au passage que son insertion au sein de l'armée est telle qu'il initiera, à la suite de sa psychologie de l'éducation, une théorie de la doctrine militaire conçue comme "communauté de conduite $»^{36}$. Voilà que sa pédagogie de la doctrine fondée sur l'ancrage " inconscient » débouche inévitablement sur l'endoctrinement et la fameuse notion de "réflexe » du militaire en combat. Le Texte de la doctrine rejoint ici le Geste inconscient de la doxa. Mais laissons là ces considérations destinées à prendre la mesure de l'imprégnation de Le Bon dans le champ militaire pour nous centrer sur sa psychologie des foules. Les idées de Le Bon sur les foules sont trop connues, ou pas assez c'est selon, pour ne pas être parfois reprises comme «allant de soi ». Emotive, suggestive, d'une crédulité sans limites, intransigeante et radicale, la foule est une " âme collective » qui écrase la volonté individuelle, un être collectif doué d'une psychologie et d'une spiritualité archaïque : "L'homme faisant partie d'une foule cesse d'être lui-même. Sa personnalité consciente s'évanouit dans l'âme inconsciente de cette foule. Il perd tout esprit critique, toute aptitude à raisonner, et redevient un primitif. Il en a les héroïsmes, les enthousiasmes et les violences ${ }^{37}$. L'anti-socialisme farouche au fondement des orientations choisies par Le Bon va constituer le cadre idéologique dans lequel vont 
s'échafauder ses théories sur les races, les peuples, les foules et les idées. Le racisme ${ }^{38}$ et la misogynie qui l'animent vont avoir pour effet de renforcer par analogie la négativité des foules, accentuant leur « désir » de soumission ce qui finira par leur donner pour trait de caractère principal l'obéissance servile aux meneurs. Les auteurs dont il s'est beaucoup inspiré, dont Gabriel Tarde ${ }^{39}$, vont vivement critiquer ses positions en soulignant l'amalgame fait entre tous les types de rassemblements. Tarde rappelait également, comme Freud le fera plus tard, qu'il convient de distinguer les foules "naturelles", rassemblées de façon spontanée, des foules "artificielles", groupes organisés, structurés, intégrés dans la durée. Mais ces distinctions, à la base de toute recherche dans ce domaine, ne seront jamais insérées dans le discours militaire y compris contemporain. En revanche, les différentes figures imaginairesque peut prendre la foule, chez Le Bon mais aussi chez Canetti, réapparaissent régulièrement dans les propos des militaires spécialisés en la matière. A l'origine, la personnalisation de la foule lui attribuant un « corps » et un " esprit $»^{40}$ permet de transposer différents stéréotypes récurrents dans la pratique du contrôle des foules. Un des principes de base étant de maîtriser "l'esprit " de la foule en isolant les personnes "non grata", en neutralisant les meneurs tout en usant de sa force de persuasion. Le "corps » de la foule peut ensuite faire l'objet d'un traitement visant à le "canaliser" ou à le «disperser». Le traitement militaire des foules, dans la continuité du traitement politique dont elles ont fait l'objet au début $\mathrm{du} \mathrm{XX}^{\mathrm{ème}}$ siècle, trouve chez Le Bon et d'autres des analogies suffisamment fortes pour les saisir - et mieux s'en saisir - par une image.

Bien sur, le bref aperçu qui suit sur les différentes figures fantasmées que peuvent prendre les foules n'est en rien restrictif ou exclusif. Suivant les situations et les acteurs engagés, ces images, dont la fonction est d'ouvrir sur le plan psychique des espaces de projection (et non d'identification), seront plus ou moins prégnantes et peuvent se superposer ou s'effacer derrière d'autres représentations. Les visages que peut prendre la foule sont autant d'images véhiculées par des valeurs culturelles très marquées historiquement. On peut schématiquement les résumer par trois termes: le Fou, la Femme et le Délinquant ${ }^{41}$. Le Fou, effigie de la maladie mentale, figure en premier lieu puisque l'annihilation de la conscience des individus composant la foule entraîne immanquablement une pathologisation de tous les comportements en foule et de la foule. Les techniques mises en œuvre pour contrôler les foules consisteront alors à adopter une position cherchant à tout prix à contenir les débordements ou les " crises ", usant pour ce faire de techniques de contention que l'on peut assimiler à un dispositif (barrières, couloirs, opposition physique, etc.) d'assujettissement du " corps » (pour reprendre l'expression foucaldienne) de la foule visant à «dominer» le malade. La Femme ensuite parce qu'elle est frivole et facilement influençable, émotive et intuitive, incapable de produire un raisonnement logique qui soit comparable à celui de l'homme. Cette misogynie revendiquée de Le Bon trouve parfois un terrain favorable encore aujourd'hui chez certains militaires surinvestissant l'identité masculine attachée à leur profession. La féminité est souvent mise en avant pour adopter une attitude de séduction, d'influence communicationnelle dont le seul but est "d'impressionner " (c'est aussi le sens des "parades » militaires). Ces deux premières figures vont pouvoir fusionner pour donner la foule hystérique, assimilation fréquemment reprise dans le maintien de l'ordre comme ailleurs. Enfin, on peut retrouver la trace du Délinquant ${ }^{42}$ dans les descriptions qu'Hippolyte Taine a fait des foules révolutionnaires dans son monumental ouvrage: Les Origines de la France 
contemporaine $^{43}$, lesquelles ont fortement influencé Le Bon et marqué toute la psychologie des foules.

Une des significations imaginaires sociales les plus partagées, au-delà même du champ militaire, s'enracine en effet dans la dévalorisation sociale culturellement très ancrée des populations qui constituent la majorité des foules: «population » prend ici son sens le plus négatif de "populace». Ce sont les gens les plus marginalisés qui forment le noyau des foules, ces gens, pour le dire sans ambages, que l'on répudie comme la « lie » de la société, autant d'a priori que nous avons choisi, en raison de sa dimension criminalisante, de résumer sous le terme de "délinquant». Le maintien de l'ordre autant que le contrôle des foules qui en est issu, entretient ses légendes comme toute activité sociale de pouvoir qui se respecte et qui entend se faire respecter. En voici une des plus significatives qui illustre de façon exemplaire notre propos : «Lors des émeutes de 1848, un officier reçut l'ordre de faire évacuer la place en tirant sur la 'canaille'. Il fit prendre position à ses soldats qui mirent la foule en joue. Dans le silence profond qui s'établit alors, il s'écria : 'j'ai reçu l'ordre de tirer sur la canaille, mais comme j'aperçois devant moi beaucoup d'honnêtes gens, je leur demande de se retirer pour que je puisse exécuter cet ordre' en quelques minutes la place se trouva vide $~_{44}$. En dépit du fait que l'anecdote résume expressément comment la foule disparaît lorsque les éléments délinquants ou supposés tels en sont écartés à l'aide, soulignons le, d'une communication particulièrement habile, efficace, comme est censée l'être la persuasion en pareil cas, cette petite scène, factuelle ou non, nous ramène inévitablement à ce qui constitue le principe même du contrôle des foules : la dispersion.

Le but ultime de toute action dite de "contrôle » est en effet, en cela le maintien de l'ordre et le contrôle des foules peuvent diverger, de provoquer la dispersion. L'endiguement et le refoulement ${ }^{45}$ peuvent être recherchés mais uniquement de façon secondaire ou intermédiaire. La priorité reste la dissolution de la masse. Si les techniques de base provenant des pratiques progressivement misent en œuvre par les forces de maintien de l'ordre à l'intérieur du territoire, telle que l'ouverture laissée à l'arrière pour le reflux ou la fuite (ne pas encercler une foule), sont sensiblement les mêmes dans l'armée de terre, d'autres techniques ne le sont pas ou ne peuvent pas l'être : Le principe déterminant de la symbolisation de la force, par exemple, n'est pas retenu alors que celui de la réversibilité immédiate de la force ${ }^{46}$ est mis en avant pour permettre à tout moment le basculement (de façon symétrique à la foule qui peut à tout moment basculer dans la violence) dans une configuration guerrière où le "contrôle " des foules devient clairement "attaque» des foules. La réversibilité immédiate empêche toute symbolisation de la force parce que celle-ci doit toujours être présente comme riposte et menace.

17 La violence est totalement maîtrisée dans la mesure où il n'y a plus de risque de débordements ou d'explosions. S'il faut pour cela détruire la source de cette violence, la foule devra donc disparaitre, par dispersion ou par éclatement. Une autre figure imaginaire peut alors symboliquement prendre place au cœur de ce combat de l'armée contre la violence des foules: l'ennemi, d'autant que cette figure essentielle, constitutive de l'imaginaire militaire, tend peu à peu à s'effacer. "Sur le terrain d'opération, les forces d'intervention doivent faire face à une combinaison de violences, induisant une érosion de la notion d'ennemi $»^{47}$. Déjà reconnue comme adversaire potentiel $^{48}$, la foule peut, sur le plan des significations imaginaires, prendre la place de l'ennemi mais ce ne peut être qu'un ennemi fictif, pour ne pas dire étrange, d'une 
étrangeté inquiétante. En effet, les jeux symboliques auxquels se livre l'armée souvent à son insu à travers les puissantes images associées à la foule ressorties du substrat imaginaire qui nourrit ces représentations, posent un problème beaucoup plus radical.

Obstacle avons-nous dit dans l'engagement des forces armées, les populations ainsi désignées comme foules, particulièrement lorsqu'il s'agit d'opérations de rétablissement de la paix, doivent être protégées et peuvent néanmoins devenir une menace. Or les actions menées dans le strict cadre défini par la loi et la doctrine militaire française visent à disperser toutes les foules, à les dissoudre, autant dire à les éliminer. Bien entendu, il doit y avoir le moins de victime possible mais comment protéger les civils tout en combattant la foule? S'il est vrai que le discours institué sur les foules, leur attribuant une essence violente purement destructrice, accentue la part d'irrationnel ou d'inconscient (dans le sens de non conscient) supposé présent dans tout rassemblement humain qui devient une "foule", et met donc l'accent sur ce qui échappe fondamentalement à toute maîtrise, le contrôle des foules peut bien se retrouver dans le paradoxe de vouloir contrôler ce qui par nature est incontrôlable. Cette foule, chargée de toute la négativité possible tient lieu d'autre différent de l'ennemi, une altérité radicale contre laquelle bute (l'obstacle est de taille) l'armée. « La foule est le contraire d'une armée: une assemblée d'hommes que ne gouverne plus rien, sinon l'humeur immédiate, le développement d'émotions passagères et contagieuses qui nuisent à l'intérêt général ». Cette citation de John Keegan, portée en exergue d'un article de la revue Objectif Doctrine ${ }^{49}$ vient étayer notre constat: la foule est bien l'antithèse de l'armée, dans l'imaginaire doctrinal, c'est pourquoi elle paraît d'emblée comme dépourvue d'organisation, de fonctionnalité, de positivité. Sa seule organisation ne peut être que psychologique. Mais nous avons vu en quoi cet imaginaire construit sur la "foule psychologique» ne peut voir l'opposition fondamentale, qui condamne par avance tout concept monolithique de foule, entre les deux pôles structurels, les deux formes antinomiques de foules telles qu'elles ont été définies par Tarde puis par Freud: la foule dite primaire, "naturelle», spontanée, dénuée de structure profonde, et la foule dite secondaire, "artificielle", très organisée, structurée de façon rigide, hiérarchique. La contradiction rendue possible par la nouvelle orientation doctrinale uniformisée sous l'expression « contrôle des foules » va dans le sens de l'exacerbation de cette polarité pour prendre un tour radical. Le nœud du problème inhérent au contrôle des foules se situe dans ce face à face entre deux foules antinomiques : d'une part l'armée, la foule « artificielle » par excellence ${ }^{50}$ et de l'autre la foule perçue comme "naturelle ». Cette dernière étant étrangère à l'armée, adversaire ou ennemie mais surtout antithèse, le contrôle des foules ne peut s'extraire de cette opposition, qui est aussi conflit, pour penser les rapports de l'armée à la foule en terme de processus englobant - pour comprendre le rapport dialectique qui unit les deux - qui intégrerait les interactions et les influences réciproques à partir desquelles se construit l'identité de chacune de ces deux entités. Si l'on se place dans cette configuration qui relativise les positions et introduit de la réciprocité, il apparaît que la réduction à l'état de "foule" de toutes les manifestations ou mouvements collectifs oblitère immanquablement les significations sociales, historiques et politiques que ces mouvements peuvent porter ou créer. Les pratiques y compris discursives liées au contrôle des foules risquent alors de dénier toute force positive à la «foule " et d'hypothéquer durablement la capacité instituante de mouvements sociaux émergeants ou en devenir 


\section{NOTES}

1. Williams R., Culture and society, New-York, Columbia University Press, 1958, p. 300.

2. Ce bref liminaire renvoie à l'hypothèse formulée différemment par Freud : «Les masses comme l'individu gardent sous forme de traces mnésiques les impressions du passé », in Moïse et le monothéisme, collection Idées, Paris, Gallimard, 1948, p.127.

3. Le terme de propagande, au-delà des techniques qui en sont l'expression formelle, doit bien entendu être pris dans le sens de la propagande moderne, en se rapportant par exemple à la définition qu'en a donné Lasswell : «La propagande est le langage destiné à la masse », (Lasswell H. D., in Propaganda, communication and public opinion, Princeton, 1946) cité par Jean-Marie Domenach dans La propagande politique, Paris, Presses Universitaires de France, 1973, p. 8.

4. Le décret-loi du 23 octobre 1935 a fixé la première réglementation des manifestations en France. Sur cette question de l'institutionnalisation des manifestations, voir les analyses produites dans l'ouvrage publié sous la direction de Pierre Favre, La Manifestation, Paris, Presses de la Fondation Nationale de Sciences Politiques, 1990.

5. Contrairement à Eugène Enriquez qui emploie cette formule pour désigner la masse manipulée ou la « foule solitaire » (in De la horde à l'Etat, Paris, Gallimard, 1983, p. 67), nous voulons signifier ici que la foule est simplement " sans nom d'auteur ", dans tous les sens du mot « auteur».

6. Commandement de la doctrine et de l'enseignement militaire supérieur de l'armée de terre, L'action des forces terrestres au contact des réalités. Une nouvelle approche doctrinale, document CDES, 2000.

7. Auteur anonyme, «Le contrôle des populations urbaines, Quels modes d'actions? Avec quels moyens?", Casoar, avril 2001.

8. TTA 950, Manuel provisoire d'emploi des forces terrestres dans le contrôle des foules, Approuvé par lettre $n^{\circ} 0866 /$ DEF/ EMAT/ BCSF/ CB du 8 août 2001.

9. « La foule, pacifique ou non, représente toujours un réservoir potentiel de violence, dont l'énergie forte peut apparaître très vite et constituer un réel danger ", TTA 950, op. cit.

10. A ce sujet, voir Beauchard J., La puissance des foules, éditions des Presses Universitaires de France, 1985.

11. La foule se définit d'abord par le nombre indéterminé des membres censés en faire parti. En cela elle est avant tout multitude et l'on sait combien le problème de la multitude (ainsi que celui, bien que différent, de la masse) est un problème éminemment politique. Sur la question du nombre et la dimension politique des foules, voir l'article de Dominique Reynié, «Théories du nombre ", ainsi que celui de Jean-Pierre Chrétien-Goni, "La mise à mort des masses ", dans la revue Hermès, $n^{\circ} 2$, Paris, éditions du CNRS, 1988. Dans une toute autre perspective, on peut également consulter de Hélène L'Heuillet, «'La dernière souveraine de l'âge moderne' A propos de la Psychologie des foules de Gustave Le Bon », in La célibataire, printemps 2003, pp. 33- 43.

12. Sur la peur des foules, voir Barrows S., Distorting mirrors, visions of the crowd in Late NineteenthCentury France, Yale University, 1981. Traduction française sous le titre, Miroirs déformants, Paris, Aubier, 1990. Voir aussi Moscovici S., L'Age des foules, Paris, Fayard, 1981, réédité aux éditions Complexe, Bruxelles, 1991.

13. Castoriadis C., L'institution imaginaire de la société,Paris, Seuil, 1975, p. 141.

14. Sur le caractère non scientifique de la notion de foule, voir l'article de Thiec Y.,.Tréanton J.R, « La foule comme objet de science », Revue française de sociologie, 24, 1983, pp. 119-136.

15. La psychologie des foules, dont le promoteur fut Gustave Le Bon, a été construite par des criminologues entre la France et l'Italie à la fin du 19ème siècle. Ces principaux auteurs ont été Tarde, Sighele, Fournial, et plus tard Freud. 
16. Ansart P., Idéologies, conflits et pouvoir, Paris, Presses Universitaires de France, collection Sociologie d'aujourd'hui, 1977, pp. 21-22.

17. Ibid.

18. Castoriadis C., «Imagination, imaginaire, réflexion », repris dans Fait et à faire, Les carrefours du labyrinthe V, collection La couleur des idées, Paris, Seuil, 1997, p. 228.

19. Castoriadis C., op. cit., p. 196.

20. "Les significations imaginaires sociales ne doivent pas être confondues avec les divers types de significations ou de sens à partir desquels Max Weber essayait de penser la société. (...) Les significations imaginaires sociales sont ce par quoi de telles visées subjectives, concrètes ou "moyennes", sont rendues possibles ", Castoriadis C., L'institution imaginaire de la société, op. cit.,p. 490.

21. Voir les publications de Loup Francart et notamment son ouvrage, Maîtriser la violence. Une option stratégique, Paris, Economica, 1999.

22. Commandement de la doctrine et de l'enseignement militaire supérieur de l'armée de terre, Les forces terrestres au contact des réalités. Une nouvelle approche doctrinale, document CDES.

23. Se reporter au paragraphe sur La nature de la foule, sa matérialisation et son dessein dans le TTA 950, op. cit., et plus précisément p. 36.

24. Le Bon G., Psychologie des foules (1895), Paris, Presses Universitaires de France, réédition de 1995.

25. Voir Périès G., « Populo-politico-militaire: un mot à l'aube de la Vème République ", Mots, $\mathrm{n}^{\circ} 55$, juin 1998.

26. En même temps qu'elle peut sembler «matérialiser » la population à un moment donné, la foule peut tout aussi bien « représenter » celle-ci dans les discours journalistiques ou politiques.

27. Voir Francart L., Maîtriser la violence. Une option stratégique, Paris, Economica, 1999.

28. La typologie des masses retenue dans le TTA 950, op. cit., reprend certaines dénominations de Canetti : tel est la cas pour les «masses de fuite». Voir Canetti E., Masse et puissance, Paris, Gallimard, 1966.

29. Loup Francart, dans son ouvrage déjà cité, reprend la description des foules de Le Bon sans le citer p. 231: «La foule se comporte alors comme une véritable entité psychologique: sous l'emprise d'émotions fortes, ses sentiments sont toujours simplistes et exagérés; elle devient alors intolérante, irritable, susceptible, impulsive et ne supporte aucun délai entre son désir et la réalisation de celui-ci », in Francart L., Maîtriser la violence. Une option stratégique, Paris, Economica, 1999.

30. Bien entendu, l'histoire de cet héritage et de sa traduction dans les pratiques et les discours politiques, policiers et militaires de manipulation et de contrôle des foules n'est certainement pas linéaire, le va et vient entre les expériences françaises et anglaises ou américaines de "crowd control » en témoignent, une telle histoire nécessiterait notamment de s'arrêter longuement sur cette étape importante pour l'armée française que fut la guerre d'Algérie.

31. Dont Mussolini qui enverra une lettre félicitant Le Bon, Hitler qui s'inspirera de ses thèses pour écrire Mein Kampf, mais aussi de nombreux militaires français dont le général de Gaulle. A ce sujet, voir Moscovici S., L'Age des foules, Complexe, Bruxelles, 1991 ; et Sternhell Z., La droite révolutionnaire, Paris, Gallimard, 1997.

32. Sur la réception de Gustave Le Bon dans l'armée française, voir Nye R.A., The origins of crowd psychology: Gustave Le Bon and the crisis of mass democracy in the Third Republic, Londres, Sage Publications, 1975 ; et Marpeau B., Gustave Le Bon. Parcours d'un intellectuel, Paris éditions du CNRS, 2000.

33. Y compris par les phrénologues dont il reprendra les travaux pour tenter de démontrer l'inégalité des races grâce aux différentes mesures du crâne humain.

34. Le Bon G., La psychologie politique et la défense sociale, Paris, Flammarion, 1910, p. 116.

35. Le Bon G., ibid., p. 93. Dans sa Psychologie de l'éducation, Le Bon cite l'ouvrage du commandant d'état-major Gaucher : Etude sur la psychologie de la troupe et du commandement, et fait référence au 
grand-duc Constantin Constantinovich, grand maître des Ecoles militaires de Russie qui a traduit son ouvrage.

36. " Elle représente le fruit d'une éducation spéciale, forcément très longue. Ses effets ne se produisent que lorsqu'elle est arrivée à ancrer certaines notions dans l'inconscient de tous les officiers d'une armée. Alors seulement, ces derniers envisagent avec une même optique mentale les situations les plus inopinées et s'y comportent, par conséquent, de façon identique ", Le Bon G., Psychologie politique et défense sociale, op. cit., p. 97.

37. Le Bon G., Aphorismes du temps présent, Paris, Flammarion, 1913, cet aphorisme reprend les idées que Le Bon développe dans sa Psychologie des foules, Paris, Presses Universitaires de France, 1995, p.14. Il ne s'agit pas d'étudier ici ces thèses qui ont fait l'objet de nombreux commentaires. A ce sujet, voir Moscovici S., L'Age des foules, Bruxelles, Complexe, 1991 ; et Marpeau B., Gustave Le Bon, Parcours d'un intellectuel, Paris, CNRS éditions, 2000.

38. Le racialisme de Le Bon, théorie naturaliste et évolutionniste qui affirme l'inégalité entre les races, est sans conteste raciste envers les peuples dits " primitifs » et de façon particulièrement outrageante (et détestable) envers les Juifs. Sur l'antisémitisme de Le Bon, voir son article sur le Rôle des juifs dans la civilisation, publié dans la Revue Scientifique en 1888, et réédité par Les Amis de Gustave Le Bon, Paris, 1989.

39. Tarde G., L'opinion et la foule, (1901), Paris, Presses Universitaires de France, 1989.

40. On retrouve ce dualisme simpliste dans les écrits de doctrine : «Le renseignement devra porter entre autre sur 'l'état d'esprit' de la foule », TTA 950, op. cit., p. 74. Cette «psychologie » des foules se résume le plus souvent en une interprétation des émotions des corps des individus en foule consistant à attribuer un sentiment ou une idée commune à des personnes uniquement sur l'observation d'un comportement identique supposé " collectif ». Sur cette question, voir Mariot N., «Les formes élémentaires de l'effervescence collective ou l'état d'esprit prêté aux foules ", Revue française de science politique, vol. 51, n5, octobre 2001, p. 707-738.

41. Sur les figures de l'imaginaire des foules, voir l'ouvrage de Susanna Barrows, op. cit.

42. Ou « déviant » souvent associé à la figure de l'alcoolique.

43. Taine H., Les origines de la France contemporaine, Paris, Hachette, 1876-74, six volumes. Ces descriptions sont largement démenties par les recherches de George Rudé. Voir Rudé G., The Crowd in the French Revolution, Oxford, Oxford University Press, 1959, traduction française sous le titre La foule dans la Révolution Française, Paris, Maspero, 1982.

44. Repris dans l'ouvrage de Marc E. et Picard D., L'Ecole de Palo Alto, Retz, Paris, 2000, p. 101.

45. TTA 950, op. cit., p. 112-113.

46. Voir sous la direction d'Emmanuel-Pierre Guittet, Cercle sur les nouvelles perspectives sécuritaires dans les doctrines française, britannique et allemande, Rapport pour la Délégation aux Affaires Stratégiques, 2002.

47. Commandement de la doctrine et de l'enseignement militaire supérieur de l'armée, op. cit.

48. TTA 950, op. cit., p.35.

49. Direction des Etudes et de la Prospective de l'Ecole d'Application de l'Infanterie, «L'infanterie dans le contrôle des foules ", Objectif Doctrine, $n^{\circ} 30$, février 2002, p. 18.

50. Rappelons que Freud prenait l'armée, avec l'église, comme exemple typique de foule secondaire dans son étude « Massenpsychologie und Ich-Analyse », traduit sous le titre " Psychologie des foules et analyse du Moi », dans Essais de psychanalyse, Paris, Payot, 1981. Voir « Nature de la foule et de l'organisation », in Enriquez E., De la horde à l'Etat, Paris, Gallimard, 1983, pp. 61-73. 


\section{RÉSUMÉS}

La fin de la Guerre Froide et la redéfinition des enjeux stratégiques qui s'en est suivi a vu le resurgissement des «foules" dans la doctrine militaire française à travers la pratique du " contrôle des foules ». Le recours à la notion de "foule » signe le retour de l'imaginaire que charrie le mot, depuis la Psychologie des foules de Gustave Le Bon. L'objet de cet article est d'élucider les significations imaginaires sociales, selon la terminologie de Castoriadis, à l'œuvre dans les pratiques et le discours des militaires français autour des «foules» et de leur " contrôle », en s'appuyant notamment de la lecture du Manuel de la Doctrine de l'armée de terre française sur le sujet.

The end of the Cold War and the redefinition of strategic stakes that followed were the occasion of the return of the notion of « crowds » in the French military doctrine troughout the action of " crowd control». This signs the return of the imaginary accompanying the word « crowd » since Gustave Lebon's work. Through the reading of the French military doctrine manual, this article aims at elucidating the imaginary social significations, according to C. Catoriadis, that are part of the French army discourses and practices with regards to the « control » of « crowds ».

INDEX

Mots-clés : Doctrine militaire, France, contrôle des foules, discours

\section{AUTEUR}

\section{ELWIS POTIER}

Elwis POTIERest psychosociologue et formateur en sciences humaines. 